\title{
Strong late-time circumstellar interaction in the peculiar supernova iPTF14hls
}

\author{
Jennifer E. Andrews` and Nathan Smith \\ Steward Observatory, University of Arizona, 933 North Cherry Avenue, Tucson, AZ 85721, USA
}

Accepted 2018 February 28. Received 2018 February 27; in original form 2017 December 1

\begin{abstract}
We present a moderate-resolution spectrum of the peculiar Type II supernova (SN) iPTF14hls taken on day 1153 after discovery. This spectrum reveals the clear signature of shock interaction with dense circumstellar material (CSM). We suggest that this CSM interaction may be an important clue for understanding the extremely unusual photometric and spectroscopic evolution seen over the first $600 \mathrm{~d}$ of iPTF14hls. The late-time spectrum shows a doublepeaked intermediate-width $\mathrm{H} \alpha$ line indicative of expansion speeds around $1000 \mathrm{~km} \mathrm{~s}^{-1}$, with the double-peaked shape hinting at a disc-like geometry in the CSM. If the CSM were highly asymmetric, perhaps in a disc or torus that was ejected from the star 3-6 yr prior to explosion, the CSM interaction could have been overrun and hidden below the SN ejecta photosphere from a wide range of viewing angles. In that case, CSM interaction luminosity would have been thermalized well below the photosphere, potentially sustaining the high luminosity without exhibiting the traditional observational signatures of strong CSM interaction (narrow $\mathrm{H} \alpha$ emission and X-rays). Variations in density structure of the CSM could account for the multiple rebrightenings of the light curve. We propose that a canonical $1 \times 10^{51} \mathrm{erg}$ explosion energy with enveloped CSM interaction as seen in some recent SNe, rather than an entirely new explosion mechanism, may be adequate to explain the peculiar evolution of iPTF14hls.
\end{abstract}

Key words: circumstellar matter - supernovae: general - supernovae: individual: iPTF14hls stars: winds, outflows.

\section{INTRODUCTION}

The very peculiar Type II supernova (SN) iPTF14hls was a so far unique event. It had a nearly unchanging and fairly normal spectrum resembling a traditional Type II-P explosion, but this was difficult to reconcile with its highly unusual multipeaked light curve, which also exhibited a high luminosity lasting far longer than any normal SNe II-P (Arcavi et al. 2017). The optical light curve had a bumpy plateau lasting over $600 \mathrm{~d}$ with five separate brightening episodes, and the velocities in optical spectra evolved 5-10 times slower than a normal SN II-P. Due to the lack of narrow lines of hydrogen typically seen in interacting Type II SNe (i.e. SNe IIn) and the absence of any detectable X-ray or radio emission, Arcavi et al. (2017) argued against shock interaction with dense circumstellar material (CSM) as the cause for the strangeness of this SN (although they did not definitively rule it out). Instead it was suggested that some new evolutionary path or explosion mechanism may need to be invoked to explain the unusual properties of iPTF14hls (Arcavi et al. 2017).

Based on a number of recent studies of SNe IIn, CSM interaction is understood to be a pathway to get long lasting, high-luminosity, and irregular or bumpy light curves. Through CSM interaction, kinetic energy of the fast SN ejecta is converted to luminosity in a dense radiative shock when the SN ejecta slams into the CSM (for a recent review of interacting SNe; see Smith 2016). Dense shells of CSM can generate extremely high luminosity in some $\mathrm{SNe}$ (Smith \& McCray 2007; Woosley, Blinnikov \& Heger 2007), and some SNe IIn can be powered this way for a long time (Rest et al. 2011) - much longer than conventional emission from a recombination photosphere that fades roughly on a diffusion time-scale (Arnett 1996). Since the luminosity generated by CSM interaction depends on the density of the CSM it overtakes, density inhomogeneities in the CSM can easily lead to irregular bumpy light curves. For instance, Nyholm et al. (2017) show that iPTF13z had a similar bumpy light curve, but its spectrum was that of a Type IIn with strong narrow emission lines. CSM interaction would seem to be a perfectly suitable explanation for the light curve of iPTF14hls, were it not for the fact that this SN's spectrum showed no signatures of CSM interaction in spectra taken over the first $600 \mathrm{~d}$ (Arcavi et al. 2017).

If we were restricted to only scenarios that permitted spherical symmetry, then the lack of narrow lines and X-rays in iPTF14hls would indeed seem to rule out CSM interaction as a plausible explanation. However, a diverse range of observations dictate that close 
binary interaction (Sana et al. 2012; Moe \& Di Stefano 2017) and asymmetric CSM (Smith 2014) are the norm among massive stars, not the exception. Binary interaction may often lead to asymmetric or disc-like CSM, especially in cases of eruptive pre-SN mass-loss (Smith \& Arnett 2014). In such cases, the situation becomes more complicated; over most of the solid angle of the explosion, the fast $\mathrm{SN}$ ejecta expand unimpeded as though there is no CSM. In the equator, however, strong CSM interaction occurs as the SN ejecta are quickly decelerated by the dense CSM. As such, the slower CSM interaction region (and hence, the CSM interaction luminosity and most observable signatures) can be quickly overrun by the rapidly expanding opaque ionized SN ejecta envelope. If the CSM interaction region is buried below the $\mathrm{SN}$ ejecta photosphere, then the luminosity created through the interaction is reprocessed by the opaque SN ejecta. From most viewing angles off the equator, this might result in a normal Type II-P spectrum that lasts until the SN ejecta opacity declines. This sort of evolution has been invoked already in PTF11iqb (Smith et al. 2015) as well as SN 1998S, SN 2009ip, and SN 1993J as discussed in Smith (2016, and references therein), where this process is referred as enveloped or swallowed CSM interaction. The main condition for enveloped CSM interaction is that the CSM is highly asymmetric. While the narrow and intermediate-width lines can be hidden partly or fully early on, they tend to emerge again at late times when the SN fades and the photosphere recedes. When this behaviour is seen, it suggests that enveloped CSM interaction may have been contributing to the luminosity the whole time. In principle, this mechanism could occur in a wide range of SN subtypes, including SNe Ia-CSM (see Smith 2016).

In this paper, we show that indeed iPTF14hls has now revealed signs of strong CSM interaction in optical spectra taken over $3 \mathrm{yr}$ after explosion, and we discuss how this scenario may provide a solution to the otherwise puzzling light curve and spectral evolution first presented by Arcavi et al. (2017). The paper is structured as follows: In Section 2, we present our new observations, in Section 3 we discuss and interpret these results, and in Section 4 we make some concluding remarks.

\section{OBSERVATIONS}

We obtained $3 \times 1200$ s exposures of iPTF14hls with the Blue Channel spectrograph on the Multiple Mirror Telescope (MMT) on 2017 November 20 UTC (JD 2458077.9). Adopting the date of discovery (JD 2456922) as day 0, following Arcavi et al. (2017), the date of our new spectrum corresponds to day 1153. The observations were taken with the $12001 \mathrm{~mm}^{-1}$ grating with a central wavelength of $6300 \AA$ and a 1".0 slit width. Seeing was 0'.9. The spectral range covers approximately 5700-7000 $\AA$ as seen in Fig. 1, and the resolving power $(R=\lambda / \delta \lambda)$ is $\sim 4500$, or $\sim 65 \mathrm{~km} \mathrm{~s}^{-1}$. Standard reductions were carried out using $\operatorname{IRAF}^{1}$ and wavelength solutions were determined using internal $\mathrm{HeNeAr}$ arc lamps. Flux calibration was achieved using spectrophotometric standards at a similar airmass taken throughout the night.

A portion of the combined 2D spectrum which has been bias and flat-field corrected but not sky subtracted is shown in Fig. 2. Both the intermediate-width and narrow $\mathrm{H} \alpha$ emission regions are

\footnotetext{
${ }^{1}$ IRAF, the Image Reduction and Analysis Facility, is distributed by the National Optical Astronomy Observatory, which is operated by the Association of Universities for Research in Astronomy (AURA) under cooperative agreement with the National Science Foundation (NSF).
}

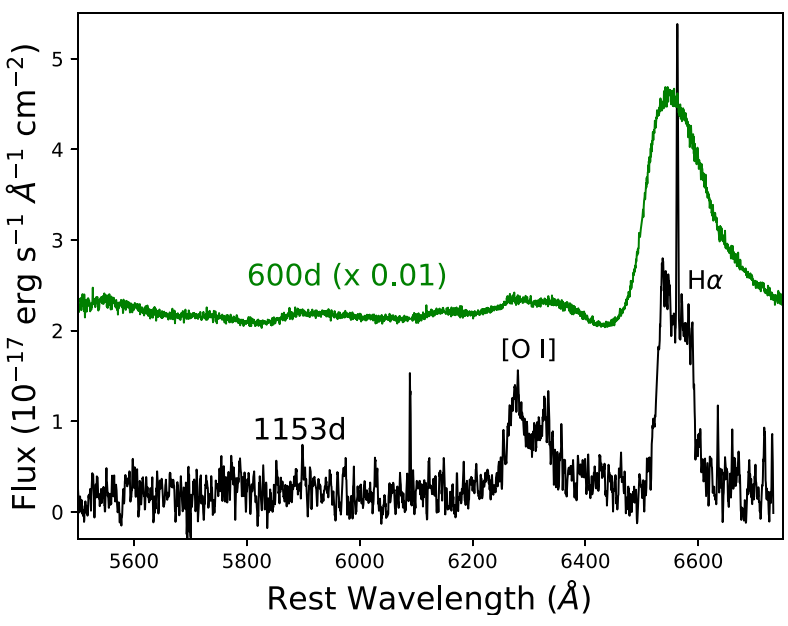

Figure 1. Day 1153 (black) and day 600 (green) spectra of iPTF14hls. Both have been corrected for redshift and extinction as discussed in Section 2. The day 600 spectrum has also been shifted by a constant for clarity and is from Arcavi et al. (2017), made available via WISeREP (Yaron \& Gal-Yam 2012).

clearly seen. The narrow $\mathrm{H} \alpha$ emission is spatially unresolved and coincident with the $\mathrm{SN}$; while we cannot rule out a local $\mathrm{H}$ II region at the $\mathrm{SN}$ position, this narrow emission is not due to spatially extended $\mathrm{H}$ II region emission in the host. If an extended $\mathrm{H}$ II region was present, we would expect to see other emission lines such as [NII] and [S II]. Additionally, an unresolved $\mathrm{H}_{\text {II }}$ region would have been seen at earlier times in previous spectra, whereas narrow $\mathrm{H} \alpha$ arising from unshocked CSM could be hidden below the photosphere and not be visible until later times when the photosophere recedes.

Following Arcavi et al. (2017), we assume negligible host galaxy extinction and correct only for Milky Way extinction of $E(B-V)=0.0137$ (Schlafly \& Finkbeiner 2011). We adopt a redshift of $z=0.0333$ for the $\mathrm{SN}$, determined from the narrow $\mathrm{H} \alpha$ emission (Fig. 3, top). This corresponds to a luminosity distance of $151 \mathrm{Mpc}$ (Planck Collaboration XIII 2016).

\section{DISCUSSION}

\subsection{Line evolution}

Fig. 1 shows the clear differences in $\mathrm{H} \alpha$ and [O $\mathrm{I}$ ] line profiles between day 600 and day 1153. Not only has the broad $\mathrm{H} \alpha$ emission narrowed, but it has taken on a boxy asymmetrical shape, and a strong narrow emission feature has emerged. The [O $\mathrm{O}$ ] $\lambda \lambda 6300,6363 \AA$ line is also much more pronounced on day 1153 than on day 600 .

Inspection of the region around $\mathrm{H} \alpha$ in the top panel of Fig. 3 shows that the velocity width of $\mathrm{H} \alpha$ has slowed from more than $6000 \mathrm{~km} \mathrm{~s}^{-1}$ (day 600) to $3000 \mathrm{~km} \mathrm{~s}^{-1}$ (day 1153). Structure has also appeared in the once smooth broad line, with two intermediatewidth bumps on the blue and red side of the narrow emission. The line profile on day 1153 can be approximated by a narrow $\left[\right.$ FWHM (full width at half-maximum) $=65 \mathrm{~km} \mathrm{~s}^{-1}$ ] Lorentzian at zero velocity plus two Guassians centred at -1000 and $700 \mathrm{~km} \mathrm{~s}^{-1}$, with FWHM values of 1380 and $1440 \mathrm{~km} \mathrm{~s}^{-1}$, respectively (Table 1). The wings of the narrow emission extend beyond $65 \mathrm{~km} \mathrm{~s}^{-1}$ and a faint narrow absorption component is seen around $80 \mathrm{~km} \mathrm{~s}^{-1}$, which is the value we adopt for the CSM velocity. The total $\mathrm{H} \alpha$ luminosity is approximately $4 \times 10^{39} \mathrm{erg} \mathrm{s}^{-1}$, at least 2 orders of 


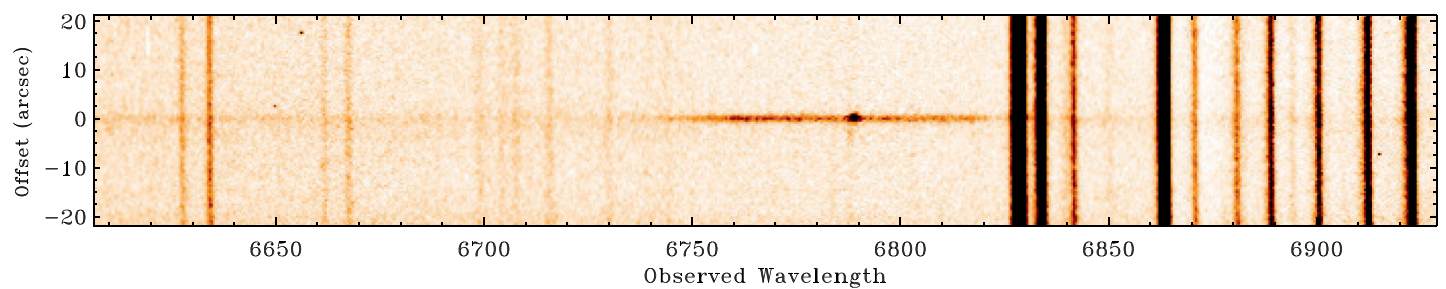

Figure 2. Stacked 2D spectrum of iPTF14hls taken on day 1153 after discovery, before subtraction of sky or host galaxy light. The intermediate-width H $\alpha$ emission is clearly seen, as is the region of the narrow $\mathrm{H} \alpha$ emission. There is some very faint extended emission to the lower side of the SN that could come from nearby $\mathrm{H}$ II regions, but it is at a different velocity from that of the narrow $\mathrm{H} \alpha$ emission in the $\mathrm{SN}$ spectrum.
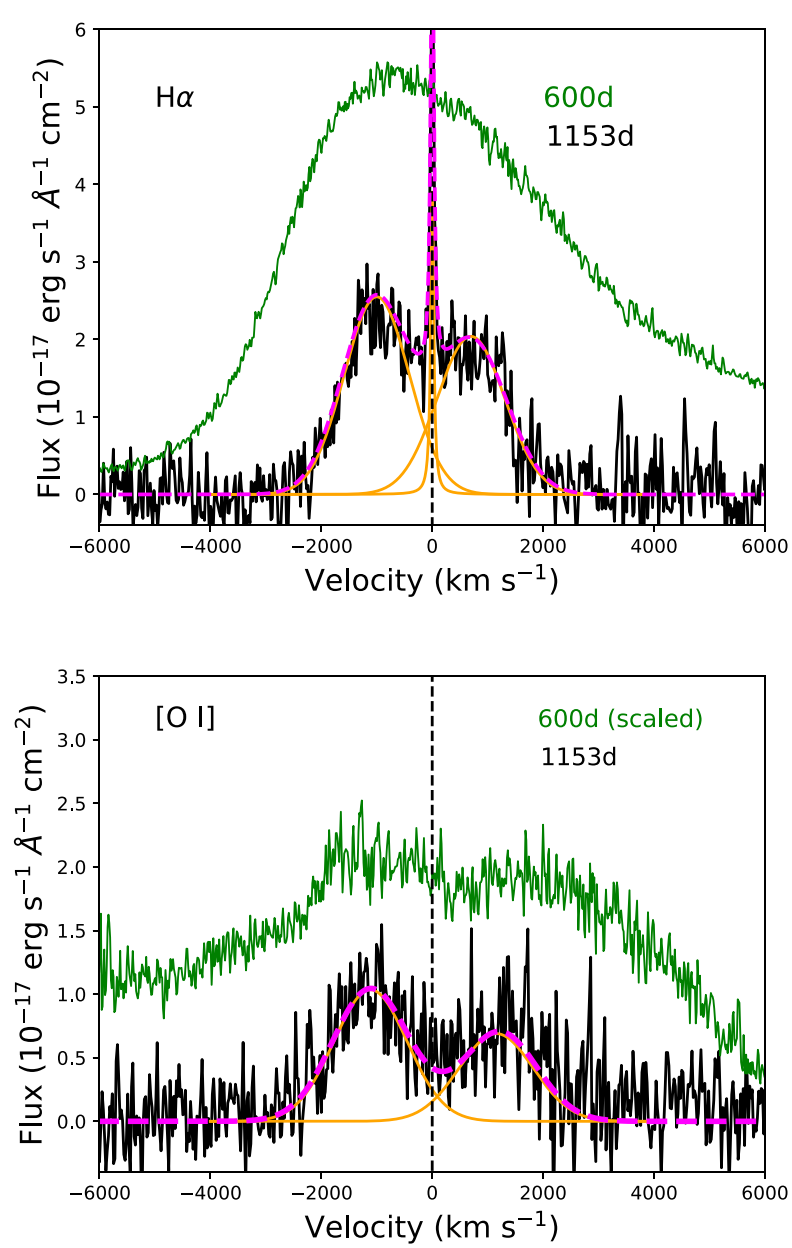

Figure 3. Velocity profiles of $\mathrm{H} \alpha$ (top) and [O I] (bottom) on days 600 and 1153. The individual component fits described in Table 1 are shown in orange and the composite in magenta, compared to the day 1153 spectrum.

Table 1. FWHM and centres of Emission Components (in $\mathrm{km} \mathrm{s}^{-1}$ ) for iPTF14hls on day 1153.

\begin{tabular}{lccc}
\hline Line & $\begin{array}{c}\text { FWHM } \\
\left(\mathrm{km} \mathrm{s}^{-1}\right)\end{array}$ & $\begin{array}{c}\text { Centre } \\
\left(\mathrm{km} \mathrm{s}^{-1}\right)\end{array}$ & $\begin{array}{c}\text { Flux } \\
\left(\mathrm{erg} \mathrm{s}^{-1} \mathrm{~cm}^{-2}\right)\end{array}$ \\
\hline $\mathrm{H} \alpha$ & 1380 & -1000 & $7.8 \times 10^{-16}$ \\
$\mathrm{H} \alpha$ & 65 & 0 & $1.3 \times 10^{-16}$ \\
$\mathrm{H} \alpha$ & 1440 & 700 & $5.8 \times 10^{-16}$ \\
{$\left[\mathrm{O}_{\mathrm{I}}\right]$} & 1550 & -1110 & $3.2 \times 10^{-16}$ \\
{$\left[\mathrm{O}_{\mathrm{I}}\right]$} & 1570 & 1150 & $2.2 \times 10^{-16}$ \\
\hline
\end{tabular}

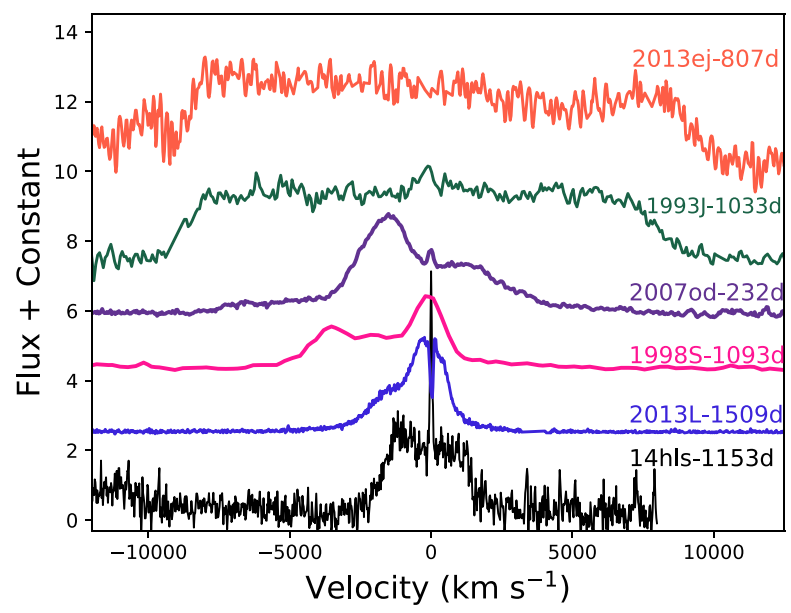

Figure 4. The day $1153 \mathrm{H} \alpha$ line of iPTF14hls compared with a few other late-time CSM interacting SNe. Data have been scaled and shifted for comparison, and come from Andrews et al. (2017, SN2013L), Pozzo et al. (2004, SN1998S), Andrews et al. (2010, SN2007od), Modjaz et al. (2014, SN1993J), and Mauerhan et al. (2017, SN2013ej).

magnitude fainter than on day 600 . Note that no corresponding photometry was taken with our spectrum to constrain the accuracy of this calibration, so this is a rough approximation.

A similar line-profile shape is seen in the [OI] emission, with the intermediate-width peaks symmetric about $6300 \AA$ and the profile becoming slightly flat-topped. This shape may have been just starting to emerge in the day 600 spectrum. The peak separation is $\sim 46 \AA$ so they are not both blue peaks from the $6300 \AA$ and $6363 \AA$ doublet. We should also see peaks at $6340 \AA$ and $6386 \AA$ if the $6364 \AA$ line behaves similarly. It is possible they are present, but as the ratio of $6300 / 6364$ is usually $3: 1$, they could be lost in the noise of the spectrum. Asymmetric oxygen profiles of this type are discussed in Milisavljevic et al. (2010), and could be due to dust either scattering internally or obscuring the red peak. The stronger blue peak in $\mathrm{H} \alpha$ could also suggest the presence of dust.

Double-peaked profiles in late-time spectra are often interpreted as due to a disc or torus CSM geometry, where the red and blue peaks arise from the SN ejecta interacting with CSM material in a disc moving radially outward from the explosion, and are not due to a rotation of the disc (Leonard et al. 2000; Hoffman et al. 2008; Mauerhan et al. 2014; Smith et al. 2015; Katsuda et al. 2016; Andrews et al. 2017, for example). We strongly suspect that geometry plays an important role in the profile shape and in the qualitative behaviour of CSM interaction in this $\mathrm{SN}$, as will be discussed in further detail below.

iPTF14hls is not the first core collapse supernova (CCSN) to show hydrogen line profile shapes of this type. Fig. 4 shows the 
similarities among the late-time $\mathrm{H} \alpha$ emission of interacting $\mathrm{SNe}$ 1993J (IIb), 1998S (IIn), 2013L (IIn), 2013ej (II-P/L), 2007od (IIP), and iPTF14hls (Fransson, Lundqvist \& Chevalier 1996; Leonard et al. 2000; Matheson et al. 2000; Fransson et al. 2005; Andrews et al. 2010; Inserra et al. 2011; Andrews et al. 2017; Mauerhan et al. 2017). This shape has also been seen in other CCSNe such as the Type II-P events SN 2011ja (Andrews et al. 2016) and SN 2008jb (Prieto et al. 2012), the Type II-L SN 1980K (Fesen \& Becker 1990), and the Type IIb SN 2013df (Maeda et al. 2015). While a broad age-range is represented, the double-peaked or boxy-shaped and flat-topped profile can be attributed to interaction with a torus or shell of CSM (Chevalier \& Fransson 1994), whether it be from a forward or reverse shock. Even without a corresponding narrow component, CSM interaction can be inferred based on the intermediatewidth line shapes and velocities. One obvious discrepancy between iPTF14hls and the other SNe is the width of $\mathrm{H} \alpha$ and the velocity of the intermediate-width peaks. Both SN 2013ej and SN 1993J have widths extending more than $7000-9000 \mathrm{~km} \mathrm{~s}^{-1}$, while iPTF14hls and SN 2013L are much slower at $1000-2000 \mathrm{~km} \mathrm{~s}^{-1}$. Intermediate between the two are SN 1998S and SN 2007od, with SN 1998S showing no red-peak at all. This is a strong indication that the CSM is more massive in the $\mathrm{SNe}$ with narrower profiles, because a higher mass within a given solid angle is needed to slow SN ejecta to $1 / 10$ th of the initial velocity. This relatively high-mass CSM would also be consistent with the high luminosity observed for iPTF14hls (see below).

\subsection{A possible scenario}

It is a challenge to simultaneously explain the long-lasting (and bumpy) light curve of iPTF14hls while also accounting for the seemingly unchanging optical spectrum of a normal SN II-P. Here, we propose that both may be explained with CSM interaction, providing that a particular set of conditions are met. Most importantly, the CSM must be highly asymmetric, perhaps in a disc or torus, and of limited radial extent. As mentioned in the Introduction, and as illustrated in Fig. 5, a disc-like geometry in the CSM may allow the CSM interaction to be hidden below the photosphere after that disc is enveloped by the fast $\mathrm{SN}$ ejecta. If the region of CSM interaction is happening below the ejecta photosphere, it can be hidden for long periods of time because the sustained CSM interaction luminosity itself keeps the surrounding SN ejecta ionized and optically thick. This phenomena is explained extensively in Smith (2016) and Smith et al. (2015), and requires only that the disc or torus of material has a limited spatial extent so that it can be overrun early on by the SN photosphere. The luminosity and duration of iPTF14hls are more extreme than PTF11iqb, but the same basic principle may apply.

In the day 1153 spectrum of iPTF14hls the narrow $\mathrm{H} \alpha$ line is the result of the photoionization of the unshocked CSM, while the intermediate-width lines arise from the $\mathrm{SN}$ ejecta slamming into the slow-moving CSM, accelerating it from $\sim 100$ to $\sim 1000 \mathrm{~km} \mathrm{~s}^{-1}$. In the scenario shown in Fig. 5, the unshocked narrow line-emitting region is shown in white, the accelerated intermediate-width material is shown in black, and the shock interaction region is shown as a dotted red line. Both the narrow and intermediate-width lines can be hidden until the photosphere recedes internal to the CSM location, as is shown in Fig. 5(b). When this occurs, signatures of CSM interaction will then be observable in the SN spectrum. If all of the slowest moving CSM is swept up by the shock, only material moving at $\sim 1000 \mathrm{~km} \mathrm{~s}^{-1}$ will be observed, otherwise a narrow component may be detected. Seeing narrow lines at $\sim 3 \mathrm{yr}$ post-explosion is uncommon, but not unexpected if the CSM is
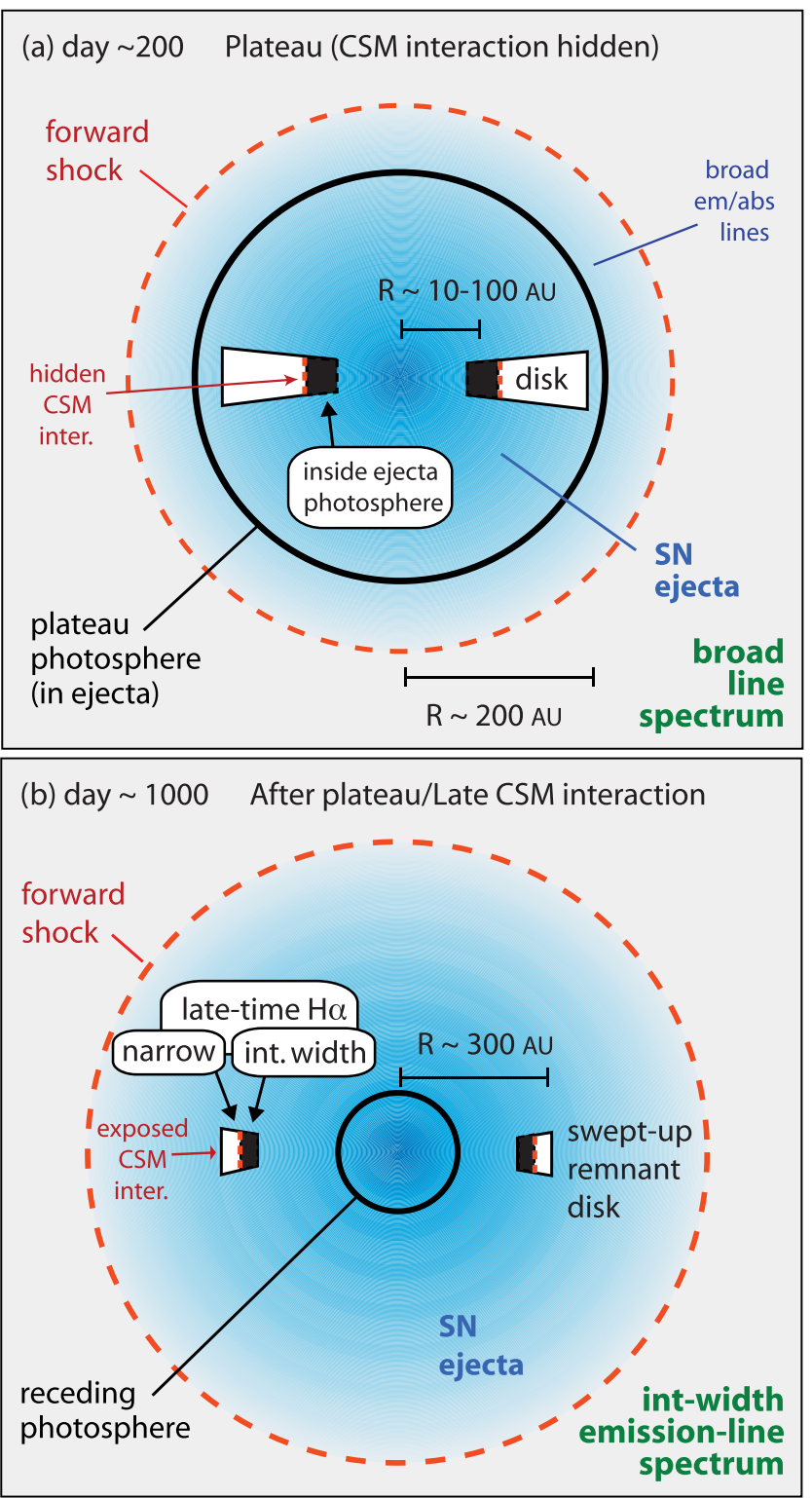

Figure 5. A sketch of enveloped CSM interaction, adapted from Smith et al. (2015), with a pre-existing disc or torus around the progenitor. (a) At early times the ejecta photosphere quickly envelops the CSM. Strong interaction occurs below the photosphere, keeping the luminosity high while hiding observational evidence that interaction is occurring. (b) At late times the photosphere has receded and the CSM is revealed again in the form of double-peaked intermediate-width spectral lines, as we observe in the latetime spectrum of iPTF14hls. Narrow emission arises in the pre-shock disc gas, which is also uncovered at late times.

asymmetric and dense, as it would take time for the shock to propagate out to the edge of the disc. It is also important to note that due to the uncertainty in explosion date of the SN by $\sim 100 \mathrm{~d}$, it is plausible that narrow emission lines were present at early times before the photosphere swallowed the CSM.

Since the disc/torus would only intercept a small fraction of the ejecta, most of the ejecta would expand freely and the ejecta photosphere would envelope the CSM quickly (Fig. 5a). For locations near the equator, the collision of the ejecta and the dense CSM produces a high luminosity of X-ray and ultraviolet (UV) photons that cannot escape due to the opacity of the surrounding SN ejecta 
and are thermalized below the SN ejecta photosphere. This causes a temporary increase in the luminosity of the SN (i.e. a 'bump') when a dense shell or clump is encountered by the shock. The continual internal energy deposition would also keep the spherically expanding ejecta photoionized, causing seemingly static or extremely slow spectral evolution until the photosphere completely recedes.

The reprocessing of CSM interaction luminosity by the surrounding ejecta not only prevents the X-rays from escaping, but also smears out any narrow line emission. This would explain why Arcavi et al. (2017) detected no X-ray emission or narrow lines. Any narrow emission emitted by pre-shock CSM in the disc would encounter multiple scatterings throughout the opaque SN ejecta and the extended $\mathrm{H} \alpha$-emitting region, effectively blurring the line structure until later times when the opacity dropped sufficiently. Therefore, any narrow line-emitting region could be easily buried within the SN ejecta, and would not be seen until late times.

Eventually the photosphere will recede (Fig. 5b), revealing the CSM interaction below. As a consequence of previous shock/CSM interaction, much of the $\sim 100 \mathrm{~km} \mathrm{~s}^{-1} \mathrm{CSM}$ will already have been swept up to speeds of $\sim 1000 \mathrm{~km} \mathrm{~s}^{-1}$, which would explain the velocity offsets of the red/blue peaks seen in iPTF14hls. Any remaining slow-moving CSM will be photoionized by the ongoing shock in the disc (red dashed line) and will be observed as narrow $\mathrm{H} \alpha$ emission. The remnant of this disc might also be cool and dense, enough to allow the growth of dust grains that could attenuate the red peak more than the blue peak. This has been seen in numerous Type II SNe, with a few shown in Fig. 4 (Leonard et al. 2000; Fox et al. 2009; Smith et al. 2009; Andrews et al. 2010, 2016).

Using the Gaussian fits shown in Table 1, we estimate the bulk of the shocked CSM is moving at $\sim 1000 \mathrm{~km} \mathrm{~s}^{-1}$. By the time the SN had achieved maximum light at day 220, this CSM would have been at a location of only $1.9 \times 10^{15} \mathrm{~cm}$ (possibly closer if the CSM is not assumed to instantly be accelerated to this velocity.) This is below the blackbody photospheric radius of $2 \times 10^{15} \mathrm{~cm}$, fully consistent with the interpretation of enveloped CSM interaction, and well below the Fe II line formation radius of $8 \times 10^{15} \mathrm{~cm}$ (shown in fig. 4 of Arcavi et al. 2017). On day 600, the intermediate-width peaks would be located at a distance of $\sim 5 \times 10^{15} \mathrm{~cm}$, outside of the blackbody photosphere, but still well within the $\mathrm{Fe}$ II line region, which has expanded to $2 \times 10^{16} \mathrm{~cm}$. At all times, the CSM is below the $\mathrm{H} \alpha$ line-forming radius, which should be between $5 \times 10^{16}$ and $6 \times 10^{16} \mathrm{~cm}$ by day 1153 assuming an $\mathrm{H} \alpha$ velocity of 4000 $6000 \mathrm{~km} \mathrm{~s}^{-1}$. This is important, because even at day $\sim 600, \mathrm{H} \alpha$ shows a broad P Cygni profile, indicating a large line scattering optical depth. Since the $\mathrm{H} \alpha$ emission from the CSM interaction is still well below the line-forming region of the broad $\mathrm{H} \alpha$ in the $\mathrm{SN}$ ejecta, $\mathrm{H} \alpha$ photons created in the CSM interaction below the $\mathrm{H} \alpha$ photosphere would be absorbed and re-emitted with the velocity of the SN ejecta, thus hiding the interaction signatures occurring within it.

Could the intermediate-width and narrow $\mathrm{H} \alpha$ observed at late times arise from the fastest ejecta crashing into a distant CSM shell that had a large inner cavity, so that CSM interaction has just turned on? That might explain the lack of CSM interaction signatures at early times (although it would provide no help in understanding the peculiar properties of the $\mathrm{SN}$ ). This is an unlikely explanation for the origin of the $\mathrm{H} \alpha$ emission we report here. If the fastest ejecta just now encountered a distant hollow shell at a large radius, then in addition to seeing narrow emission from the pre-shock gas and intermediate width emission from the shocked CSM, we would also expect to see very broad emission from the fastest ejecta that are at the reverse shock (Chevalier \& Fransson 1994). This is precisely the scenario that occurred in SN1987A, when the blast wave hit distant CSM after a delay of several years, and in that case the broad $\mathrm{H} \alpha$ emission from the reverse shock is quite strong (Smith et al. 2005). This broad emission is not seen here, indicating that the shock is not caused by the fastest ejecta intercepting a distant shell. Instead, a shock in the CSM at a much smaller radius that was previously hidden below the photosphere would have much slower SN ejecta of only $1000-2000 \mathrm{~km} \mathrm{~s}^{-1}$ catching up to the reverse shock.

The lack of polarization detected by Arcavi et al. (2017) suggests that the bulk of the continuum emission during the first year or two of the iPTF14hls light curve came from a mostly spherical environment. In a simple scenario, this might be at odds with our requirement of highly asymmetric CSM, as long as the $\mathrm{SN}$ is not viewed pole-on. However, again, in the scenario we suggest with enveloped CSM interaction, the asymmetric emitting regions are buried well below the photosphere. Since these CSM interaction photons are thermalized deep inside the $\mathrm{SN}$ ejecta envelope, their polarization signature would be erased.

The asymmetric CSM was likely created through some enhanced episodic or explosive mass-loss shortly before core-collapse. If for instance the CSM is located at a distance between 50 and 100 au $\left(10^{14.5}-10^{15} \mathrm{~cm}\right.$; this is the distance derived by extrapolating the late-time expansion speed of $1000 \mathrm{~km} \mathrm{~s}^{-1}$ to around day 100-200), the fastest narrow $\mathrm{H} \alpha$ emission velocity of $\sim 80 \mathrm{~km} \mathrm{~s}^{-1}$ (measured from the blue edge of the narrow emission line) would require a mass-loss episode sometime between 3 and 6 yr before discovery. This is in agreement with Arcavi et al. (2017) and the time-scale is consistent with late nuclear burning instabilities such as pulsational pair instabilities or wave-driven mass-loss from Ne/O burning (Quataert \& Shiode 2012; Smith \& Arnett 2014; Fuller 2017; Woosley 2017). In addition to the advanced burning instabilities, binary interaction with a bloated envelope would likely need to be invoked in order to explain the pronounced asymmetry in the CSM (Smith \& Arnett 2014).

CSM interaction is an efficient way to convert $\mathrm{SN}$ ejecta kinetic energy into radiative luminosity. Arcavi et al. (2017) estimate a total radiated energy for iPTF14hls of about $2 \times 10^{50} \mathrm{erg}$. This can easily be achieved with CSM interaction and a conventional SN explosion energy of $1-2 \times 10^{51} \mathrm{erg}$ and typical IIP ejecta mass of $10 \mathrm{M}_{\odot}$, provided that a massive disc or torus intercepts about 10-20 per cent of the solid angle of the SN ejecta (Smith, Mauerhan \& Prieto 2014). From momentum conservation, a CSM mass of 5-10 $\mathrm{M}_{\odot}$ would be required to decelerate that fraction of a typical SN ejecta mass down to the final observed coasting speed of $1000 \mathrm{~km} \mathrm{~s}^{-1}$. The ejecta mass can be increased as long as the CSM mass is also increased, provided the interaction conserves momentum and yields a final velocity of $\sim 1000 \mathrm{~km} \mathrm{~s}^{-1}$. The opening angle of the CSM can also be tweaked, as long as it is still concentrated in a disc/torus. While a 5-10 $\mathrm{M}_{\odot} \mathrm{CSM}$ mass requires rather extreme pre-SN mass-loss rates compared to normal steady winds, it is not so extreme compared to some high-luminosity SNe IIn, such as SN 2006gy, SN 2006tf, and SN2010j1 [see Smith (2014) for a review of pre-SN mass-loss]. This sort of scenario is also less extreme than the $50 \mathrm{M}_{\odot}$ or more of SN ejecta, and unusually high-explosion energy of $10^{52} \mathrm{erg}$, and perhaps multiple explosions, that is required in a scenario without CSM interaction (Arcavi et al. 2017).

\section{CONCLUSIONS}

While the evolution of iPTF14hls is quite unusual among SNe studied so far, we propose that it may be explained with an enveloped CSM interaction scenario, and without necessarily invoking an 
unprecedented explosion mechanism. With two assumptions - (1) that the SN progenitor had an extreme mass-loss episode a few years before explosion, as is generally thought to be the case for most $\mathrm{SNe}$ IIn, and (2) that binary interaction or rapid rotation caused this CSM to be distributed into a disc or torus - it may not be so challenging to explain both the observed spectrum and light curve of iPTF14hls, as well as the multipeaked and boxy emission-line profiles seen at late times. This is possible if the CSM is dense and close enough to the star that it can be overrun and enveloped by the SN ejecta. The CSM interaction region could be hidden beneath the $\mathrm{SN}$ photosphere for months or years, but its luminosity would be thermalized and would increase the emergent optical luminosity of the event by keeping the surrounding SN ejecta ionized. Viewed by an outside observer, the SN ejecta that are reheated by CSM interaction from deep inside would exhibit the spectrum of a normal, broad Type II-P event. Multiple shells or some other density inhomogeneities in the CSM could cause the emergent light curve to appear bumpy as the shock propagates through each of them. Therefore, it is plausible that the strange behaviour of iPTF14hls is due to circumstellar interaction, despite the lack of obvious signatures of CSM interaction in the early spectra.

There may indeed have been a brief period of time when iPTF14hls showed strong narrow lines and other signs of CSM interaction, but because the first spectra were obtained around $100 \mathrm{~d}$ after explosion, these might have already faded when the CSM interaction region was enveloped by the opaque fast $\mathrm{SN}$ ejecta. Additional observations can help constrain the mass-loss history of the progenitor and further uncover the interaction occurring in iPTF14hls. For example, although early time X-ray and radio observations yielded non-detections, the current interaction seen in $\mathrm{H} \alpha$ may suggest that deep late-time X-ray observations should be obtained. The IIn SN 2005ip had an X-ray luminosity $\sim 25 \times$ brighter than the $\mathrm{H} \alpha$ luminosity at late times (Smith et al. 2017). Assuming iPTF14hls behaves similarly we can estimate an $L_{X}=1 \times 10^{41} \mathrm{erg} \mathrm{s}^{-1}$. At the distance of iPTF14hls and similar assumptions about absorption as in SN 2005ip, Chandra ACIS observations with roughly an hour of on source time should readily detect this. It would also be interesting to produce 2D radiative transfer models for this asymmetric scenario of enveloped CSM interaction to see exactly which CSM geometries and viewing angles are consistent with a lack of narrow lines in iPTF14hls.

\section{ACKNOWLEDGEMENTS}

Special thanks to Jon Mauerhan for the spectrum of SN 2013ej and to the referee for constructive comments. NS acknowledges puzzling but fruitful conversations with I. Arcavi about iPTF14hls. The day 600 spectrum of iPTF14hls was obtained from the Weizmann interactive supernova data repository (http://wiserep.weizmann.ac.il) Yaron \& Gal-Yam (2012). Support was provided by NSF grants AST-131221 and AST-151559, and by a Scialog grant from the Research Corporation for Science Advancement. Observations reported here were obtained at the MMT Observatory, a joint facility of the University of Arizona and the Smithsonian Institution.

\section{REFERENCES}

Andrews J. E. et al., 2010, ApJ, 715, 541

Andrews J. E. et al., 2016, MNRAS, 457, 3241

Andrews J. E., Smith N., McCully C., Fox O. D., Valenti S., Howell D. A., 2017, MNRAS, 471, 4047

Arcavi I. et al., 2017, Nature, 551, 210

Arnett D., 1996, Supernovae and Nucleosynthesis: An Investigation of the History of Matter, from the Big Bang to the Present. Princeton Univ. Press, Princeton

Chevalier R. A., Fransson C., 1994, ApJ, 420, 268

Fesen R. A., Becker R. H., 1990, ApJ, 351, 437

Fox O. et al., 2009, ApJ, 691, 650

Fransson C., Lundqvist P., Chevalier R. A., 1996, ApJ, 461, 993

Fransson C. et al., 2005, ApJ, 622, 991

Fuller J., 2017, MNRAS, 470, 1642

Hoffman J. L., Leonard D. C., Chornock R., Filippenko A. V., Barth A. J., Matheson T., 2008, ApJ, 688, 1186

Inserra C. et al., 2011, MNRAS, 417, 261

Katsuda S. et al., 2016, ApJ, 832, 194

Leonard D. C., Filippenko A. V., Barth A. J., Matheson T., 2000, ApJ, 536, 239

Maeda K. et al., 2015, ApJ, 807, 35

Matheson T., Filippenko A. V., Ho L. C., Barth A. J., Leonard D. C., 2000, AJ, 120, 1499

Mauerhan J. et al., 2014, MNRAS, 442, 1166

Mauerhan J. C. et al., 2017, ApJ, 834, 118

Milisavljevic D., Fesen R. A., Gerardy C. L., Kirshner R. P., Challis P., 2010, ApJ, 709, 1343

Modjaz M. et al., 2014, AJ, 147, 99

Moe M., Di Stefano R., 2017, ApJS, 230, 15

Nyholm A. et al., 2017, A\&A, 605, A6

Planck Collaboration XIII, 2016, A\&A, 594, A13

Pozzo M., Meikle W. P. S., Fassia A., Geballe T., Lundqvist P., Chugai N. N., Sollerman J., 2004, MNRAS, 352, 457

Prieto J. L. et al., 2012, ApJ, 745, 70

Quataert E., Shiode J., 2012, MNRAS, 423, L92

Rest A. et al., 2011, ApJ, 729, 88

Sana H. et al., 2012, Sci, 337, 444

Schlafly E. F., Finkbeiner D. P., 2011, ApJ, 737, 103

Smith N., 2014, ARA\&A, 52, 487

Smith N., 2016, in Alsabti A. W., Murdin P., eds, Interacting Supernovae: Types IIn and Ibn, Handbook of Supernovae. Springer-Verlag, Berlin, p. 403

Smith N., Arnett W. D., 2014, ApJ, 785, 82

Smith N., McCray R., 2007, ApJ, 671, L17

Smith N., Zhekov S. A., Heng K., McCray R., Morse J. A., Gladders M., 2005, ApJ, 635, L41

Smith N. et al., 2009, ApJ, 695, 1334

Smith N., Mauerhan J. C., Prieto J. L., 2014, MNRAS, 438, 1191

Smith N. et al., 2015, MNRAS, 449, 1876

Smith N. et al., 2017, MNRAS, 466, 3021

Woosley S. E., 2017, ApJ, 836, 244

Woosley S. E., Blinnikov S., Heger A., 2007, Natur, 450, 390

Yaron O., Gal-Yam A., 2012, PASP, 124, 668

This paper has been typeset from a $\mathrm{T}_{\mathrm{E}} \mathrm{X} / \mathrm{L} \mathrm{T}_{\mathrm{E}} \mathrm{X}$ file prepared by the author. 